\title{
The building of the distribution function of the normative generalized power influence on brick buildings
}

\author{
Oxana Kichaeva* \\ O.M. Beketov National University of Urban Economy in Kharkiv, 61002 Kharkiv, Ukraine
}

\begin{abstract}
Loads and impacts which have an influence on the performance of the structure have high statistical distribution, being random variables or random processes. The influence on frameless brick buildings and buildings with a mixed frame from the following types of loads and impacts is considered: load from own weight of structures, load from people and equipment (payload), snow load, wind load, external temperature effects and impacts due to ground motion (settlement). The types of buildings were considered: frameless buildings and buildings with a mixed frame. The article proposes an algorithm for constructing the distribution function of the generalized force effect on brick buildings and structures. Obtaining such a function is one of the stages of the critical normative risk (probability) of failure of brick structures of buildings. It is determined that the distribution function of the generalized force action obeys the normal distribution law of Gauss, for which the values of the mathematical expectation and the coefficient of variation are obtained.
\end{abstract}

\section{Introduction}

In the continuous operation, brick buildings and structures undergo simultaneous effect of loads and impacts of different types and intensity. The combination of impacts leads to reduction of the durability of buildings and structures. Considering the reliability of the entire "base - foundation - structure" system, it can be concluded that its level is influenced by the following parameters: 1) external loads and impacts, which include impacts from engineering-geological processes that occur at the base; 2) Strength and deformability of structural elements of a building (structure); 3) strength and deformability of the base. These parameters are not constant in time. So, the probabilistic approach to determining the level of reliability is due to the fact that all characteristics of the strength and deformation of the "base - foundation - structure" system, as well as all loads and impacts on the system are random variables or random processes.

In this article, an approach to the structure of the distribution function of the normative generalized force action on buildings and structures made of bricks is presented. This

\footnotetext{
*Corresponding author: o_kichaeva@ukr.net
} 
function is one of the stages of determining the value of the critical normative risk (probability) of failure of brick buildings.

\section{Discussion}

When assessing the reliability of brick buildings, the loads and impacts, regulated by existing engineering standards, must be taken into account. According to the type of loads, these classes of loads and impacts are distinguished:

1. The own weight of structures $F_{G}$.

2. The load from people and equipment (payload) $F_{q}$.

3. Climatic impacts ( $\operatorname{snow}$ load $F_{S}$, wind load $F_{W}$, external temperature effects $F_{T}$ ).

4. Impacts due to ground motion (settlement) $F_{D}$.

Loads depending on the variability in the time of impact are divided into constants and variables, depending on the causes of the load - the main and episodic.

The above loads and impacts are considered in this paper. Characteristic values of random natural influences (wind, snow, temperature) are often assigned as values exceeding 1 time in $T_{c}$ years. Then the possibility of occurrence of impact with an average period of recurrence $T_{c}$ years for one arbitrary taken year is $1 / T_{c}$, and the probability that an impact will never occur over the period of maintenance $T$ years, is calculated by the formula:

$$
P=\left(1-1 / T_{c}\right)^{T}
$$

By Norms of Ukrainian engineering it is established that the values of some of the considered loads and impacts (wind, temperature, snow and useful) can be exceeded once in 50 years. Thus, the annual probability of exceeding of these loads is:

$$
p_{w}=p_{T}=p_{s}=p_{q}=0.021 \text { year } .
$$

The probability of either impact will never occur over the period of maintenance $T$ years (for a period of $\mathrm{T}$ we will adopt the reference value of the life of residential and public buildings), equal to 100 years, will be:

$$
P=(1-1 / 50)^{100}=0.133 .
$$

When performing calculations for assessing the reliability of buildings made of bricks in conditions of an unevenly deformable base due to the normative method there should be considered calculated cases for the main combination of loads and impacts. In the calculated case of the basic combination, loads and impacts with normative excess probabilities corresponding to this engineering case are taken into account.

Loads influencing the structure and other external impacts are the most uncertain values and have high statistical distribution. The structure is usually effected by several loads, each of which has its own characteristics. In order to lead all these external influences to one generalized load (impact), it is necessary to understand the specific characteristics of each impact and their combinations. To determine the stock coefficient, it is desirable that the entire set of loads, randomly varied in time, is led to one random variable $\mathrm{F}$, which can be inserted into the calculation formulas.

Loads and impacts on bricks buildings and structures can be regarded as independent random variables. When performing probabilistic calculations to determine the risk of the emergence of one of the critical conditions of buildings and structures made of bricks, the laws of distribution of these loads and the impacts with respect to their variability should be 
known. On the basis of the laws of distribution of each impacts, the distribution law of the generalized force action $F$ can be obtained, which can be used to perform calculations.

The following loads and impacts have influence on brick buildings and structures, such as payload on the overlap $F_{Q}$, snow load $F_{S}$, wind load $F_{W}$, temperature impacts $F_{T}$, impacts of the uneven settlement $F_{D}$, can be considered independent random variables. The own weight $F_{G}$ of the structure can be regarded in some cases as a deterministic value due to its small variability, and in some cases as a random variable.

Below is an approach for determining the parameters of the distribution laws of loads and impacts on brick buildings and structures, as well as the parameters of the distribution law of generalized force impact. Thus, it is possible to be confined to values of standard factors and values of standard probabilities of excess of loads and impacts.

The distribution functions of random loads and impacts can be adopted in accordance with the following distribution laws:

- own weight $F_{G}$ of the structure is the normal distribution law, which is characterized by the mathematical expectation $\mu_{G}$ and the mean squared deviation $\sigma_{G}$; we will also note that the value of the structures' own weight can be assumed deterministic because of its small variability;

- payload on overlaps $F_{Q}$ - double exponential distribution law of Gumbel, which is characterized by mathematical expectation $\mu_{Q}$ and the mean squared deviation $\sigma_{Q}$;

- snow load $F_{S}$ is also the distribution law of Gumbel, which is characterized by the mathematical expectation $\mu_{S}$ and the mean squared deviation $\sigma_{S}$;

- wind load $F_{W}$ - Gumbel distribution law, which is characterized by the mathematical expectation $\mu_{W}$ and the mean squared deviation $\sigma_{W}$;

- the temperature impacts $F_{T}$ can be taken in accordance with the normal distribution law, which is characterized by the mathematical expectation $\mu_{T}$ and the mean squared deviation $\sigma_{T}$;

- the impact of uneven settlement $F_{D}$ can also be taken in accordance with the normal distribution law, which is characterized by the mathematical expectation $\mu_{D}$ and the mean squared deviation $\sigma_{D}$.

According to EN 1990 [1] and DBN B.1.2-2:2006 [2], the calculated combinations should include loads that most adversely affect the structures (bases) from the point of view of the critical state. According to EN 1990, the combinations of impacts for permanent or transitional calculated situations (basic combinations) are generally described as follows:

$$
E_{d}=\gamma_{S d} E\left\{\gamma_{G, j} G_{k, j} ; \gamma_{p} P ; \gamma_{Q, 1} Q_{k, l} ; \gamma_{Q, i} \psi_{0, i} Q_{k, i}\right\} \quad j \geq 1, i>1 .
$$

The combination of impacts must include:

- the calculated value of the leading (dominant) time impact, and

- the calculated combination of accompanying temporal effects.

According to DBN B.1.2-2:2006, combinations of two types - basic and emergency can be used in structure calculations. To test the critical states of the first group, basic combinations are used, which include constant loads with the critical calculated values, as well as critical calculated, cyclic, or quasi-constant values of variable loads. To test the marginal states of the second group, basic combinations are used, including constant loads with operational values, as well as operational design, cyclic or quasi-permanent values of variable loads. In emergency combination, in addition to permanent and variable loads, there can be one episodic value.

For basic combinations involving constant and not less than two variable loads, the latter are adopted with a combination coefficient $\psi_{1}=0.95$ for long loads and $\psi_{2}=0.90$ for short-term loads. Then the basic combination of loads can be written in the notations of EN 
1990 for the combination of the constant $G$ and the two variable loads $Q_{k, 1}$ and $Q_{k, 2}$, while for them the values are taken for both short-term loads:

$$
C_{m}=\gamma_{G}{ }^{*} G_{k}{ }^{*}+\gamma_{Q, 1}{ }^{*} \psi_{t, 1} Q_{k, 1}{ }^{*}+\gamma_{Q, 2}{ }^{*} \psi_{t, 2} Q_{k, 2}{ }^{*} .
$$

The symbols $G_{k}{ }^{*}, Q_{k, 1}{ }^{*}$ mean the normative values of the constant and variable loads. The coefficient of reliability $\gamma_{G}{ }^{*}$ (in DBN - $\gamma_{f m}$ ) for a constant load, which is meant to be the weight of structures and soils, is adopted depending on the type of material and varies within the range of $1.05 \ldots 1.3$. The reliability coefficient for a variable load is adopted depending on its type. The load reliability coefficient $\gamma_{f m}$ for evenly distributed loads should be taken: 1.3 - at a characteristic value of less than $2.0 \mathrm{kPa}\left(200 \mathrm{kgf} / \mathrm{m}^{2}\right) ; 1.2$ - with a characteristic value of $2.0 \mathrm{kPa}\left(200 \mathrm{kgf} / \mathrm{m}^{2}\right)$ and more. The reliability coefficient of responsibility (responsibility coefficient) $\gamma_{n}$ is determined depending on the class of consequences (responsibility) of the object depending on the calculated situation and the group of critical states.

The active loads and impacts on the "base - foundation - structure" system for the problems considered in this paper can be summarized as follows (Table 1). The data given in Table 1, are obtained both from the results of the normative literature and authors dealing with this subject, and from the author's own data.

Table 1. Statistical parameters of load distributions.

\begin{tabular}{|l|c|c|c|c|c|}
\hline \multicolumn{1}{|c|}{ Type of load } & $\begin{array}{c}\text { The } \\
\text { designation }\end{array}$ & Units & $\begin{array}{c}\text { Average } \\
\text { value }\end{array}$ & $\begin{array}{c}\text { Variation } \\
\text { coefficient } \\
\boldsymbol{V}\end{array}$ & $\begin{array}{c}\text { The } \\
\text { distribution } \\
\text { law }\end{array}$ \\
\hline Constant load & $G$ & $\mathrm{kN}$ & $G$ & 0.1 & Normal \\
\hline Payload (50 years) & $Q$ & $\mathrm{kN} / \mathrm{m}^{2}$ & $0.6 Q$ & 0.35 & Gumbel \\
\hline Snow load (50 years) & $S$ & $\mathrm{kN} / \mathrm{m}^{2}$ & $0.7 S_{0}$ & 0.5 & Gumbel \\
\hline Wind load & $W$ & $\mathrm{kN} / \mathrm{m}^{2}$ & $0.75 W_{0}$ & 0.35 & Gumbel \\
\hline Temperature impact & $T$ & $\mathrm{C}^{\mathrm{o}}$ & $T$ & 0.15 & Normal \\
\hline $\begin{array}{l}\text { Uneven settlement } \\
\text { impact (effort in the } \\
\text { foundation) }\end{array}$ & $D$ & $\mathrm{kPa}$ & $P$ & 0.59 & Normal \\
\hline
\end{tabular}

The distribution function of the generalized force action obeys the normal distribution law of Gauss with the mathematical expectation of $\mu_{F}$ and the variation coefficient $V_{F}$. This statement can be explained by the fact that the generalized force action, considered as a random variable, is the sum of the loads and impacts on the structure, and the loads and impacts are independent random variables. In accordance with the central critical theorem of probability theory, when summing a sufficiently large number of independent random variables, the distribution of their sum is close to normal.

\section{Results}

Knowing the values of the variation coefficients of various loads, we can determine the value of the variation coefficient of the generalized force action for both the sum of the random variables, assuming that the values of the generalized mathematical expectation $\mu_{\Sigma}$ and the mean squared deviation $\sigma_{\Sigma}$ are known.

In this case, $\mu_{\Sigma}$ is the total summary value of the mathematical expectation from various impacts, equal to:

$$
\mu_{\Sigma}=\mu_{G}+\mu_{Q}+\mu_{S}+\mu_{W}+\mu_{D} .
$$

The mean squared deviation: 


$$
\sigma_{\Sigma}=\sqrt{\sigma_{G}^{2}+\sigma_{Q}^{2}+\sigma_{S}^{2}+\sigma_{W}^{2}+\sigma_{D}^{2}} .
$$

The contribution of climatic temperature loads for the entire building (but not for a separate structure) can be neglected because of its small contribution to the generalized force impact.

Then, according to the theory of probability:

$$
\begin{gathered}
V_{\Sigma}=\frac{\mu_{\Sigma}}{\sigma_{\Sigma}}, \\
V_{\Sigma}=\sqrt{V_{G}^{2}+V_{Q}^{2}+V_{S}^{2}+V_{W}^{2}+V_{D}^{2}} .
\end{gathered}
$$

Figure 1 shows the distribution functions of the impacts distributed according to the normal distribution law with mathematical expectation equal to 1 and the corresponding variation coefficients for each type of load. The graphs were obtained using the MathCAD program.

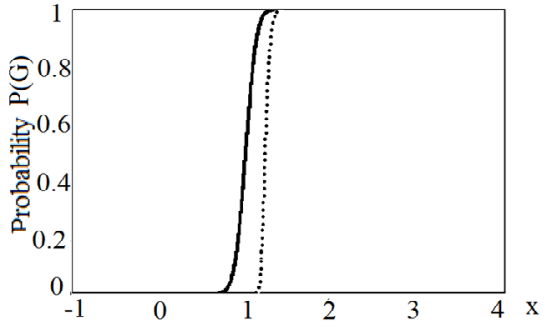

a)

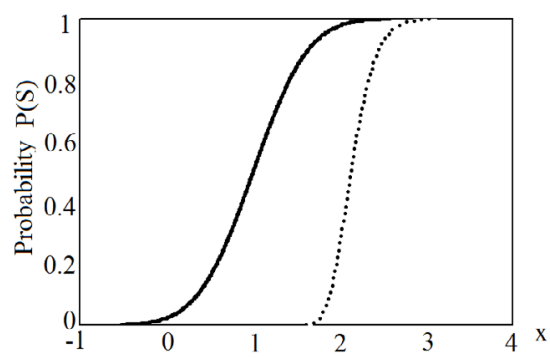

c)

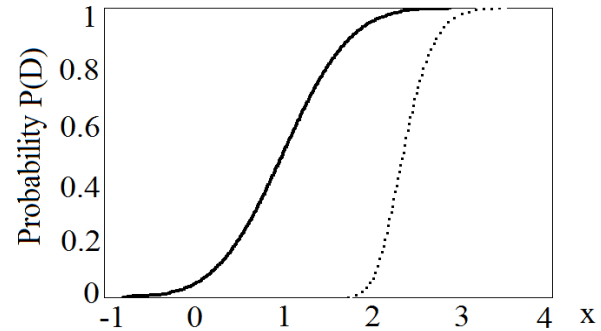

b)

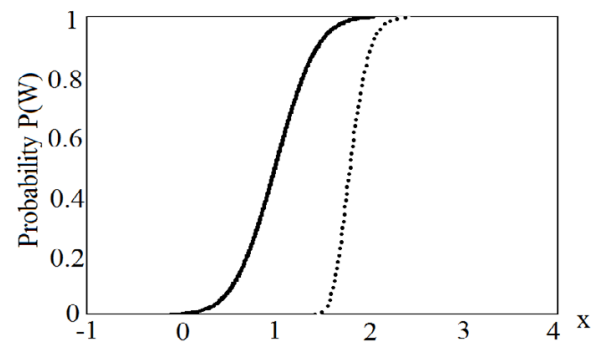

d)

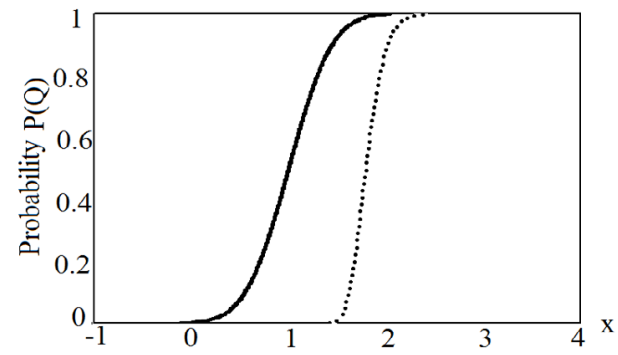

e)

Fig. 1. Graphs of the distribution function of force impacts $(x)$ : a) from own weight; b) from the influence of uneven settlement; c) from the snow load; d) from the wind load; e) from the payload. 


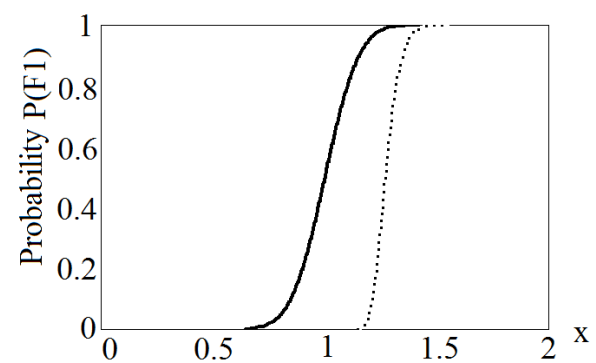

a)

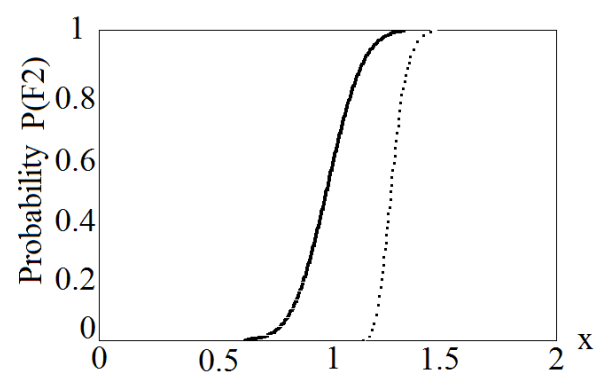

b)

Fig. 2. Graphs of distribution functions of the generalized force $(x)$ : a) for case 1 with $V_{\Sigma I}=0.119$; b) for case 2 with $V_{\Sigma 2}=0.122$.

The following variants were considered for determining $V_{\Sigma}$.

1) Frameless buildings with a cargo area of $\mathrm{A}=3 \mathrm{~m}^{2}, 6 \mathrm{~m}^{2}$ и $9 \mathrm{~m}^{2}$ with the payload value $\mathrm{q}=150 \mathrm{kgf} / \mathrm{m}^{2}, 200 \mathrm{kgf} / \mathrm{m}^{2}, 300 \mathrm{kgf} / \mathrm{m}^{2}, 5^{\text {th }}$ snow area accepted [2], $2^{\text {nd }}$ wind zone, the value of loads on foundation with its vertical displacement is accepted, with the value of deformations up to $5 \mathrm{~cm}$ inclusive, the soil deformation modulus $\mathrm{E}=8 \mathrm{MPa}, 10 \mathrm{MPa}$, $12 \mathrm{MPa}, 14 \mathrm{MPa}, 16 \mathrm{MPa}$, Poisson's coefficient is $0.2,0.3,0.4$, the square of the base of foundation varied from $1 \mathrm{~m}^{2}$ to $2 \mathrm{~m}^{2}$.

2) Buildings with a mixed frame. Parameters: $A=16 \mathrm{~m}^{2}$ with the payload value $\mathrm{q}=150 \mathrm{kgf} / \mathrm{m}^{2}, 200 \mathrm{kgf} / \mathrm{m}^{2}, 300 \mathrm{kgf} / \mathrm{m}^{2}, 5^{\text {th }}$ snow area accepted, $2^{\text {nd }}$ wind zone [2], the value of loads on foundation with its vertical displacement is accepted, with the value of deformations up to $5 \mathrm{~cm}$ inclusive, the soil deformation modulus $\mathrm{E}=8 \mathrm{MPa}, 10 \mathrm{MPa}$, $12 \mathrm{MPa}, 14 \mathrm{MPa}, 16 \mathrm{MPa}$, Poisson's coefficient is $0.2,0.3,0.4$, the square of the base of foundation varied from $1 \mathrm{~m}^{2}$ to $4 \mathrm{~m}^{2}$.

On figure 2 there are graphs of the distribution functions of the generalized force impact: a) for case 1 with $V_{\Sigma 1}=0.119$; B) for case 2 with $V_{\Sigma 2}=0.122$.

On figures 1, 2 the dashed lines show the distribution functions which represent the annual probabilities which correspond to the values of $F$, and the solid lines show the distribution functions of the impacts for $\mathrm{T}=100$ years. The value of the variation coefficient of the generalized force impact, calculated by the formula (9), equals $V_{\Sigma 1}=0.119$ and $V_{\Sigma 2}=0.122$.

\section{Conclusions}

Thus, the function of the distribution of the normative generalized force impact on buildings and structures made of bricks is constructed. With the help of this function, it is possible to determine the values of the critical normative risk (probability) of failure of brick buildings.

\section{References}

1. EN 1990:2002, Eurocode: Basis of Structural Design (Brussels, 2002)

2. DBN B.1.2-2:2006, Load and impact (Kiev, 2006) 